\title{
Simulation of prospective phytosterol intake in Germany by novel functional foods
}

\author{
Karolin Kuhlmann ${ }^{1}$, Oliver Lindtner ${ }^{2}$, Almut Bauch ${ }^{2}$, Guido Ritter ${ }^{1}$, Brigitte Woerner ${ }^{2}$ and Birgit Niemann ${ }^{2} *$ \\ ${ }^{1}$ University of Applied Sciences Münster, Department of Nutritional Sciences, Corrensstrasse 25, D-48149 Münster, Germany \\ ${ }^{2}$ Federal Institute for Risk Assessment, Nutritional Medicine, Thielallee 88-92, D-14195 Berlin, Germany
}

(Received 24 March 2004 - Revised 29 October 2004 - Accepted 1 November 2004)

\begin{abstract}
A blood cholesterol-lowering margarine containing plant sterolesters was the first functional food placed on the European food market pursuant to the regulation (EC) 258/97. In the following years nine further applicants submitted the request to add plant sterol compounds to dairy products, cheeses, bakery products, sausages, plant oils and other products. The European Scientific Committee on Food (SCF) declared a precautionary intake limit of $3 \mathrm{~g}$ plant sterols per $\mathrm{d}$ by multiple dietary sources. Using the consumption data of the German National Food Consumption Study, carried out from 1985 to 1988 with 23209 participants, we hypothetically added $0 \cdot 3-2 \mathrm{~g}$ plant sterols to usual daily servings of ten different food products, selected from the novel food applications. We calculated the prospective plant sterol intake regarding each kind of enriched food and by stepwise accumulation of different functional foods in three enrichment scenarios. Within our enrichment context we find a phytosterol intake satiation, if multiple plant sterol-enriched foods are eaten. An enrichment amount of $2 \mathrm{~g}$ plant sterols per proposed food serving size results in an intake maximum of $13 \mathrm{~g} / \mathrm{d}$.
\end{abstract}

Phytosterols: Functional ingredients: Fortification: Intake simulation: Safety assessment

In the 1990s, the idea of developing foods for improving the health-state and well-being or reducing risks of common diseases, popularised as functional foods, became a main innovative trend in food development (Milner, 2000; Roberfroid, 2000). A Concerted Action on Functional Food Science in Europe (FUFOSE) was started by the International Life Science Institute (ILSIEurope) to turn food production into a new stage of scientific quality. The resulting concepts were scrutinised in a plenary meeting held in July 1997 in Helsinki and published in 1999 in a consensus document, 'Scientific concepts of functional foods in Europe' (Diplock et al. 1999). The first products developed before and in line with this concept were different types of margarine to reduce blood cholesterol, a widely accepted marker for cardiovascular diseases (Hornstra et al. 1998; Mensink et al. 2003). The functional principle of these types of margarines was realised by adding different plant sterol compounds to traditional margarine (Gylling et al. 1995; Law, 2000; Weststrate $\&$ Meijer, 1998).

Functional foods must be safe according to all standards of food risks assessment (Diplock et al. 1999). This applies even more to functional ingredients, because adding ingredients to design functional foods allows an easy transfer of the functional principle to a range of different traditional foods. This transfer of a functional principle by transferring a bioactive ingredient to common foods tends to result in the emergence of food sets of different foodstuffs, designed for the same health purpose and containing the same active ingredient. Additionally, in order to assert health claims, each product has to contain an active dosage of the functional ingredient per typical food serving. However, this trend of health-claimed functional food development raised the safety aspect of consumers' protection against excessive consumption of bioactive compounds to a new dimension. Especially in those cases where functional ingredients are drug-like compounds with measurable effects on health-associated physiological parameters, the problem of accumulation is urgent.

Plant sterols are the structural and functional counterparts of vertebrates' cholesterol. The daily supply of phytosterols by traditional 'Western nutrition' amounts to an average of 150-400 mg per person (Scientific Committee on Food, 2002a). The ability of phytosterol compounds to reduce the level of cholesterol in blood plasma of patients with different hypercholesterolaemias as well as in healthy persons is well investigated (Hendriks et al. 1999; Wester, 1999; Ostlund et al. 2002). Hallikainen et al. (2000), Law (2000) and others (Hendriks et al. 1999; Nguyen, 1999) showed that the supply of $1 \cdot 3-2 \mathrm{~g}$ of phytosterols reduces the LDL-plasmacholesterol level by about $5-15 \%$ by inhibiting the intestinal cholesterol absorption without reducing the HDL-cholesterol. Human and animal studies have shown that plant sterol esters are non-toxic (Ayesh et al. 1999; Baker et al. 1999; Hepburn et al. 1999; Waalkens-Berendsen et al. 1999; Weststrate et al. 1999; Sanders et al. 2000), indicating that these compounds are safe. For these features, phytosterol compounds became interesting as functional ingredients for the production of food able to lower the blood cholesterol level in humans. An application for marketing a plant sterol-enriched margarine was submitted in the Netherlands in 1998 according to the requirements of regulation (EC) 258/97. The product was 
approved for retailing in the European Union by the European Commission in July 2000 according to the affirmative opinion of the former Scientific Committee on Food (SCF; Scientific Committee on Food, 2000). In the following years nine further companies submitted applications for dairy products, cheeses, bakery products, sausages, plant oils and miscellaneous products with added plant sterol compounds to design a range of cholesterol-lowering functional foods (the requests are listed in http:// www.bfr.bund.de).

The increasing number of plant sterol-enriched foods draws the attention to a potentially excessive plant sterol intake by permanent consumption of multiple cholesterol-lowering foods. As part of the safety assessment, the SCF pointed out that plant sterols and stanols (1) interfere with the absorption of carotenes, (2) lead to a small but dose-related increase of their plasma concentrations and (3) have no additional benefits at intakes higher than the active dosage of between 1 and $3 \mathrm{~g} / \mathrm{d}$. Furthermore, it cannot be excluded that high intakes might induce undesirable effects. Therefore the SCF recommended avoiding plant sterol intakes exceeding the active dosage per day. Consequently, since the number of foods that are enriched with plant sterols is increasing, additional management measures may be needed to avoid excessive intake (Scientific Committee on Food, 2002a). In this context the objective of our study was to calculate the prospective plant sterol intake by plant sterol-enriched foods in Germany and to develop an exemplary enrichment scenario to maintain the precautionary intake limit set by the SCF.

\section{Methods}

\section{Selection of foods for model-fortifications with plant sterols}

Consistent with the food categories of the novel food proposals to use plant sterols as functional ingredients, we chose ten common foods of widespread and customary consumption in Germany. The numerical codes for these foods were selected from the 'Bundeslebensmittelschlüssel (BLS II.3.)' (Klemm et al. 1999), a significant German food composition table. For each selected food item a range of product variants were pooled to determine its overall consumption. For instance, the consumption amounts of the sausage 'salami' integrate the pooled dietary data of fourteen different salami variants. All dietary data were obtained by processing the material from the public use file of the German National Food Consumption Study (NVS; public use file, managed by the University of Giessen) that had been collected in the Federal Republic of Germany from 1985 to 1988 . The study with 23209 participants was based on weighed recordings over $7 \mathrm{~d}$ for every person of a household (Adolf et al. 1995). The consumption data of the NVS are linked with the numerical codes of the food composition table. We selected our model food set by determining the percentage of users in the study population, the overall consumption amounts of the foods, the average daily consumption amount by the users and the percentage of its consumption amounts within the food categories. All data processing, including the plant sterol intake calculations, has been carried out with SPSS (SPSS Inc. Chicago, IL, USA, version 12.0.1, November 2003).

\section{Simulation method}

The prospective plant sterol intake by German consumers through the consumption of one enriched food as well as of multiple enriched foods has been calculated modifying a method described by Raulio et al. (2001). These authors simulated the intake of phytosterols by Finnish adults (g/d) replacing one to four ordinary foods in an imaginary diet by products enriched with phytosterols using the following modelling method: simulated intake of phytosterols $(\mathrm{mg} / \mathrm{d})=$ consumption of food $(\mathrm{g} / \mathrm{d}) \times$ phytosterol content in the food concerned $(\mathrm{mg} / 100 \mathrm{~g})$. For this, average food consumption figures among the users of each product were used (Raulio et al. 2001).

We modified this method by defining the hypothetical phytosterol content not per $100 \mathrm{~g}$ of the food concerned, but per proposed serving sizes of the individual foods. Additionally, we calculated the phytosterol intake for each user of one and more enriched food in the following way:

$$
S I_{\text {phyt }, k}=\sum_{i=1}^{10} \frac{A A_{\text {phyt } 1-3, i}}{P F S_{i}} \times A D C_{i, k}
$$

where $S I_{\text {phyt }}$ is the simulated intake of phytosterols $(\mathrm{g} / \mathrm{d})$; $A A_{\text {phyt } 1-3}$ is the added amount of phytosterols according to the enrichment strategies of the scenarios 1 to $3(\mathrm{~g})$; $P F S$ is the proposed food serving size (g); $A D C$ is the average daily consumption amount of a food, calculated in the German National Food Consumption Study from the consumption over $7 \mathrm{~d}(\mathrm{~g} / \mathrm{d}) ; i$ is the food index; $k$ is the user index.

The ratio of the added amount of phytosterols per proposed food serving depends on the serving size and on the added amount of phytosterols. Both parameters differ in a different way between the scenarios, as well as between the foods. For the purpose of clarity, we indicated the individual values for this quotient as factors $F_{1}$ to $F_{3}$ (see Table 2).

\section{Determining the food serving sizes}

There are no international standards for typical food portion sizes useful as an allocation basis for the enrichment strategy. The different novel food applicants also used self-appointed portion sizes in their applications. It has became apparent that these types of functional foods offer an active dosage of phytosterols in a typical portion of the carrier food. Consequently, we used a German data collection referred to as 'MONICA amounts list' (AID, 1991) to propose serving sizes for each food used in our calculations. This list was generated in the context of the WHO MONICA project Augsburg (MONitoring trends and determinants In CArdiovascular disease; Tunstall-Pedoe, 1985; Colling et al. 1989) and represents a collection of weighed amounts of ordinary food portions, for instance the weight of a thin, a medium or a thick slice of bread, a slice of hard cheese, a large or a small container of yoghurt etc. To determine characteristic consumption amounts per day, we chose one or two portions from the MONICA-amounts list, as regarding the special features of the foods concerned (see Table 1). The serving size of margarine was used from the recommendations for the plant sterol-enriched margarine 'becel pro activ', which was the solely sold plant sterol-enriched food in Germany at the beginning of our study.

\section{Simulation order and age groups}

The phytosterol intake simulations were performed at first for the consumption of every single enriched food. In a second step we calculated the phytosterol intake by multiple enriched foods, replacing the original foods stepwise by their plant sterolcontaining counterparts. The cumulative intake simulation was 
Table 1. Consumption data and proposed portion sizes for the selected food items*

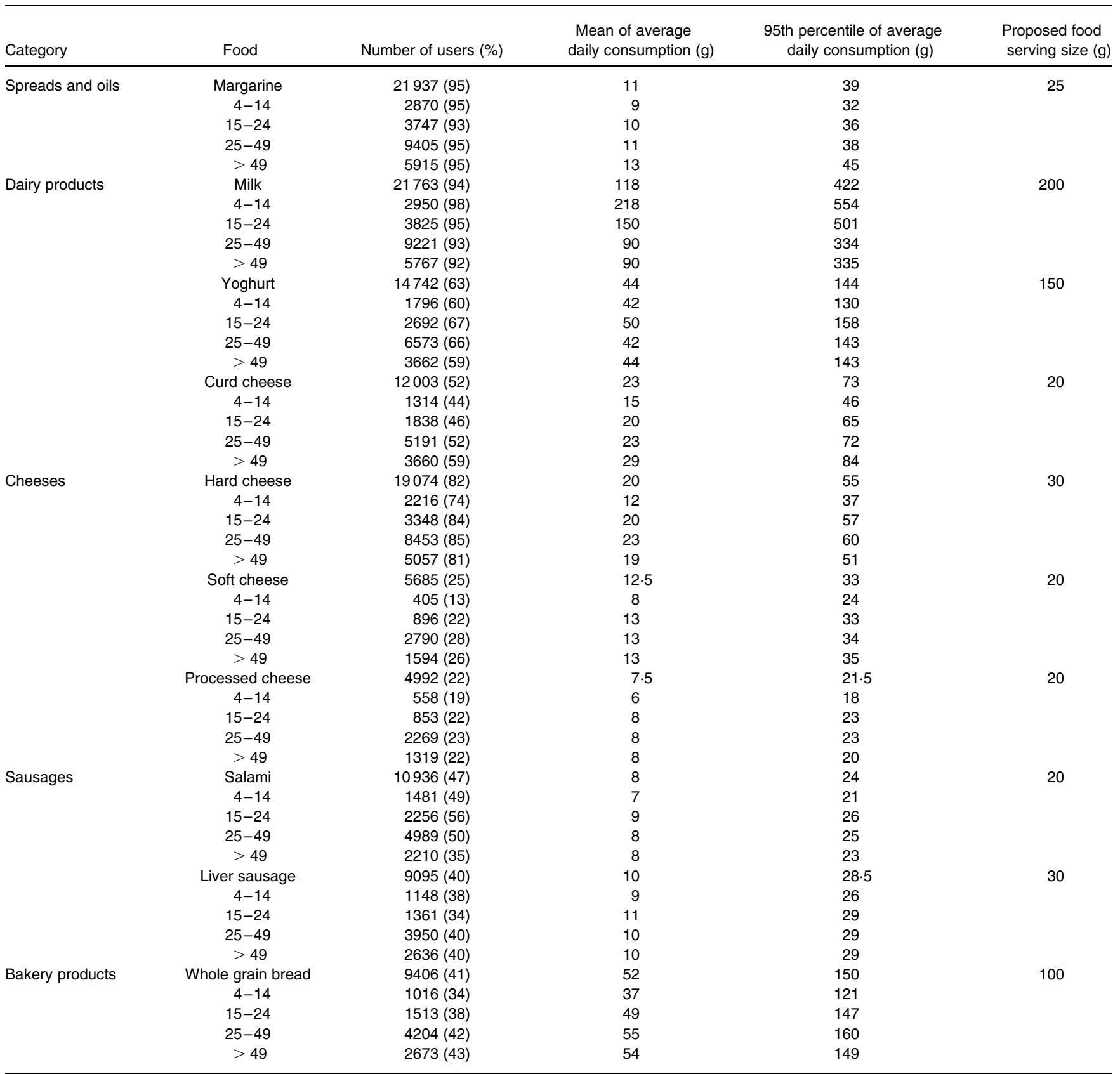

* The number of users of the selected food items, the means and the 95th percentiles of the average daily consumption amounts of the users concerned are given. In addition, the user of each food as well as the consumption data were broken down into the defined age groups. The percentages in parentheses are either related to the whole study population or in the case of the data for the age groups, to all users inside the respective age group. The proposed food serving size for margarine is equivalent to plant sterol margarine, and is the producer's recommendation. The serving sizes for milk and yoghurt conform to the amounts which are included in one glass of milk and one small container of yoghurt. For the bread $100 \mathrm{~g}$ are equivalent to two medium slices and the serving sizes for the fillings conform to one slice of bread.

ranked according to the ascending ratio between the proposed food serving size and the daily consumption amount of the respective food at the 95th consumption percentile of the users concerned. The daily intake of plant sterols from natural sources was neglected because of lack of composition data on plant sterols in the food composition table. Considering that the cholesterol-lowering claim of plant sterols is primarily aimed at elderly people with elevated blood cholesterol levels, we separated the study population into four age cohorts, where necessary. All people older than 49 years $(n$ 6250) were summarised as the potential target group and all people younger than 50 years were subdivided into the group of children (4-14 years, $n 3012$ ), the group of adolescents and young adults (15-25 years, $n$ 4008) and the group of adults (25-49 years, $n$ 9939). Infants, younger than 4 years, were not considered in the NVS.

\section{Plant sterol enrichment scenarios}

In the first scenario it was assumed that all selected food types are enriched with an effective dosage of $2 \mathrm{~g}$ plant sterols per proposed 
food serving. The added amount of $2 \mathrm{~g}$ allows the use of the cholesterol-lowering claim by producers but raises the risk of excessive plant sterol intake by consumers. In a second scenario, the recommended intake limit of $3 \mathrm{~g}$ plant sterols per day was distributed to all selected foodstuff, which resulted in a concentration of $0.3 \mathrm{~g}$ per proposed food serving. We anticipated that this second scenario would be in good compliance with the safety recommendations on the entire intake of phytosterols by multiple sources (Scientific Committee on Food, 2002a). However, in this second scenario the added amount of phytosterols per food product was not sufficient to legitimate the cholesterol-lowering claim for the individual products. Compromising these contrasts, in a third scenario we restricted the number of foods to six, which were enriched with active dosages between 1 and $2 \mathrm{~g}$ plant sterols per proposed serving. We involved the age group-specific phytosterol intake results of the first scenario in the decision on the restriction of the food items which should be enriched in scenario 3 . The consumption of milk by children and adolescents is significantly higher compared with the group of the elderly. Therefore milk was excluded in the third enrichment scenario. In addition, the enrichment of whole grain bread and of two cheese varieties (hard cheese and soft cheese) were also excluded. The exclusion of bread is not determined by substantial intake differences, but rather by considerations regarding the regular and simultaneous use of bread and spreads, maybe both in an enriched version. Similarly, two cheese varieties (hard cheese and soft cheese) were excluded in favour of the diversity of carrier foods for a phytosterol enrichment. So we chose margarine, yoghurt, two different sausages and two different cheeses for the limited third scenario. We favoured the number of six enriched foods, because this would be easy to perceive for consumers. Although this decision is reasonable, it was an arbitrary one. However, we could not find a scientific tool to determine the transition of a concise number of enriched products in a huddle.

\section{Results}

\section{Selected foods and consumption data}

The simulations described above were done for products from the food categories: spreads and oils, dairy products, cheeses, sausages and bakery products. From these categories ten food items were selected (see Table 1). The relative high number of dairy products in the food set (milk, yoghurt, curd cheese and three further cheese varieties) reflect the high number of novel food proposals to add phytosterols to dairy products. Salami was the most frequently eaten raw sausage and the same applies to liver sausage among the cooked sausages. Whole grain bread was selected from bakery products due to its healthy image. For all food items, the number and the age pattern of users within the study population, the mean of the average daily consumption amounts of the users of a food concerned as well as the 95th consumption percentiles are given in Table 1 .

\section{Prospective plant sterol intake by the consumption of one enriched food}

The amounts of phytosterols hypothetically added to one serving of the selected foods are shown in Table 2. The statistical distributions of the phytosterol intake by consuming only one enriched food is given in Fig. 1(a)-(c) for all foods in all scenarios. The small range between the level of active dosages ( $\geq 1 \mathrm{~g} / \mathrm{d})$ and the recommended intake limit $(\leq 3 \mathrm{~g} / \mathrm{d})$ is marked by bold lines. Provided that the consumers completely replace one original food by its enriched counterpart, a predominant part of the users would not reach an active daily dosage of phytosterols in all three scenarios, with the exception of eating curd cheese containing $2 \mathrm{~g}$ phytosterols in $20 \mathrm{~g}$ curd cheese. The consumption of only one enriched food like margarine, milk, hard cheese, whole corn bread or soft cheese does not provide more than $20-40 \%$ of the respective users with an effective plant sterol dosage per day. In spite of this, many consumers of each food type would get more than $3 \mathrm{~g}$ plant sterols per day. As expected, in the second scenario the risk of excessive intake is minor but the cholesterol-lowering effect should be unverifiable. In the third scenario the number of consumers with a plant sterol intake above $3 \mathrm{~g} / \mathrm{d}$ is reduced compared to the first scenario but many more users would also have an ineffective intake.

The mean phytosterol intake amounts of the four age groups (not shown) according to the enrichment strategy of the first scenario mirror the age-related consumption amounts for the foods concerned and show a distinct preference for milk and a disregard for curd cheese by children and adolescents compared to the target group of the elderly.

\section{Prospective plant sterol intake by the consumption of multiple enriched foods}

The stepwise cumulation of the prospective plant sterol intake by multiple enriched food sources in the first and the second scenario

Table 2. The enrichment strategies for the three scenarios*

\begin{tabular}{|c|c|c|c|c|c|c|}
\hline \multirow[b]{2}{*}{ Food } & \multicolumn{2}{|c|}{ Scenario 1} & \multicolumn{2}{|c|}{ Scenario 2} & \multicolumn{2}{|c|}{ Scenario 3} \\
\hline & Added amounts (g) & $\mathrm{F}_{1}$ & Added amounts (g) & $\mathrm{F}_{2}$ & Added amounts (g) & $\mathrm{F}_{3}$ \\
\hline Margarine & 2 & 0.08 & 0.3 & 0.012 & 2 & 0.08 \\
\hline Milk & 2 & 0.01 & 0.3 & 0.0015 & - & - \\
\hline Yoghurt & 2 & 0.133 & 0.3 & 0.002 & 1.5 & 0.01 \\
\hline Curd cheese & 2 & 0.1 & 0.3 & 0.015 & 1.5 & 0.075 \\
\hline Hard cheese & 2 & 0.066 & 0.3 & 0.01 & - & - \\
\hline Soft cheese & 2 & 0.1 & 0.3 & 0.015 & - & - \\
\hline Processed cheese & 2 & 0.1 & 0.3 & 0.015 & 1 & 0.05 \\
\hline Salami & 2 & 0.01 & 0.3 & 0.015 & 1.5 & 0.075 \\
\hline Liver sausage & 2 & 0.066 & 0.3 & 0.01 & 1 & 0.033 \\
\hline Whole grain bread & 2 & 0.02 & 0.3 & 0.003 & - & - \\
\hline
\end{tabular}

\footnotetext{
* The added amounts of phytosterols per proposed food serving and the specific values for this ratio $\left(F_{1}, F_{2}\right.$ and $\left.F_{3}\right)$ are given.
} 
(a)

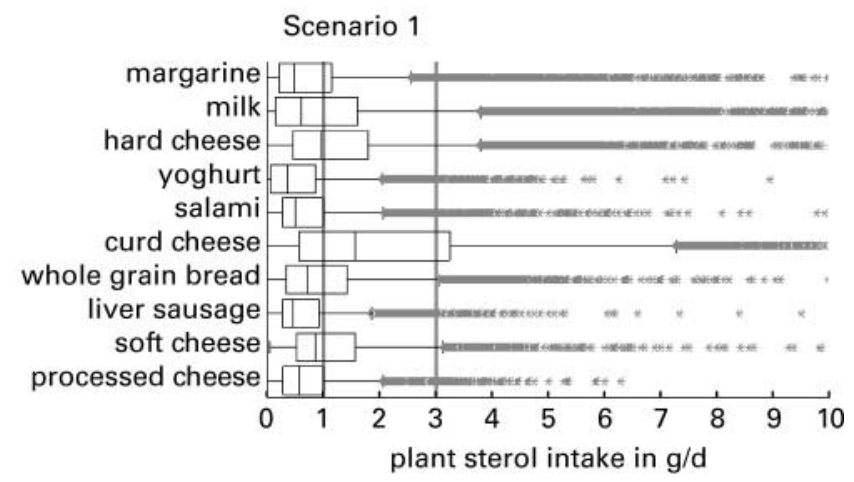

(b)

Scenario 2

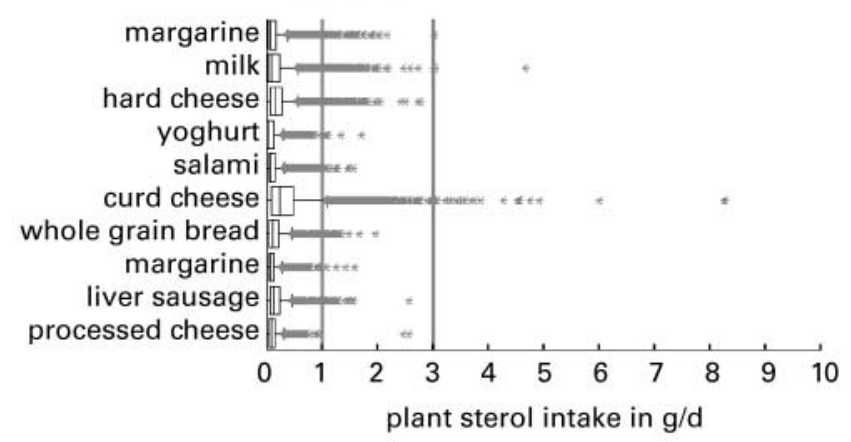

(c)

\section{Scenario 3}

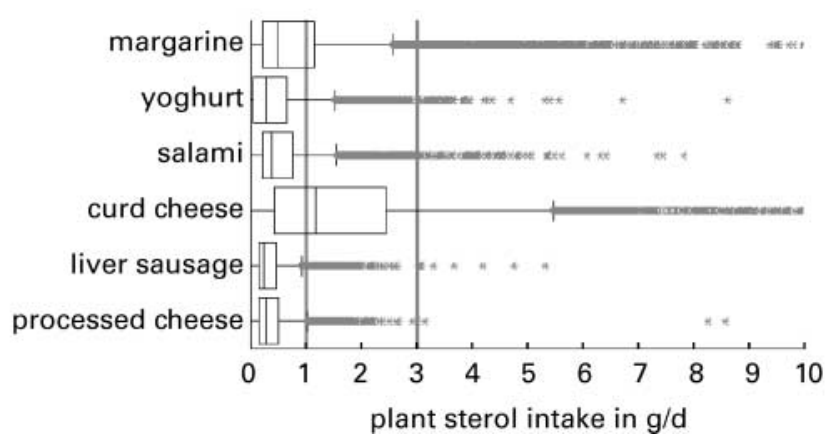

Fig. 1. Distribution of plant sterol intake by every single cholesterol-lowering food according to the enrichment strategies of the first scenario (a), the second scenario (b) and the third scenario (c). Inside the boxes are $50 \%$ of the users from the 25th to 75th intake percentile and the median values are shown as black lines. The lines outside the boxes mark the 90 th percentile. The range of the active dosage level $(\geq 1 \mathrm{~g} / \mathrm{d})$ and the precautionary intake limit $(\leq 3 \mathrm{~g} / \mathrm{d})$ are limited by bold lines. Outsider and extreme values are marked by asterisks.

has been carried out in the following ranking: curd cheese, milk, hard cheese, margarine, whole grain bred, soft cheese, salami, yoghurt, liver sausage and processed cheese. In the third scenario milk, hard cheese, whole grain bread and soft cheese were eleminated, resulting in the ranking: curd cheese, margarine, salami, yoghurt, liver sausage and processed cheese.

Fig. 2 shows the mean and the 95th percentile measurements of plant sterol intake for all cumulation steps as taken from statistical data. The chosen cumulation order follows a plausible saturation curve, indicating their suitability as worst case scenario. The maximum intake at the satiation level increased proportionally with the added amounts of plant sterols in the individual products. In our model the added amount of $2 \mathrm{~g}$ plant sterols to each food resulted in an intake maximum around $13 \mathrm{~g}$ per day at the 95th consumption percentile.
Furthermore, our calculations show that if three foods enriched with $2 \mathrm{~g}$ plant sterols per food serving size (scenario 1 ) are eaten, the mean intake will exceed the maximum limit of $3 \mathrm{~g}$, whereas in scenarios 2 and 3, the cumulative intake of all foods will not exceed this maximum mean intake limit. This applies also to the 95th percentile intake for scenario 2 . In the third scenario, when the number of enriched foods was limited to six and the added amount of plant sterols varied around the active dosage, the intake maximum of plant sterols reduced to $6 \mathrm{~g} / \mathrm{d}$. Furthermore, the users at the mean intake level were already provided with an active dosage of plant sterols with the consumption of two enriched foods (Fig. 2), whereas the users with an undesirable intake of above $3 \mathrm{~g}$ plant sterols per day decreased to $25 \%$ as compared to $80 \%$ in scenario 1 (Fig. 3(a)). In Fig. 3(b) we show the age pattern within the part of users eating more 
(a)

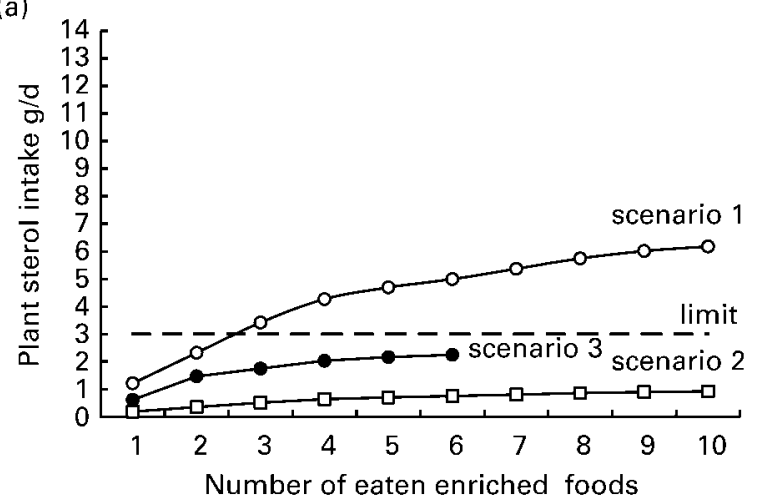

(b)

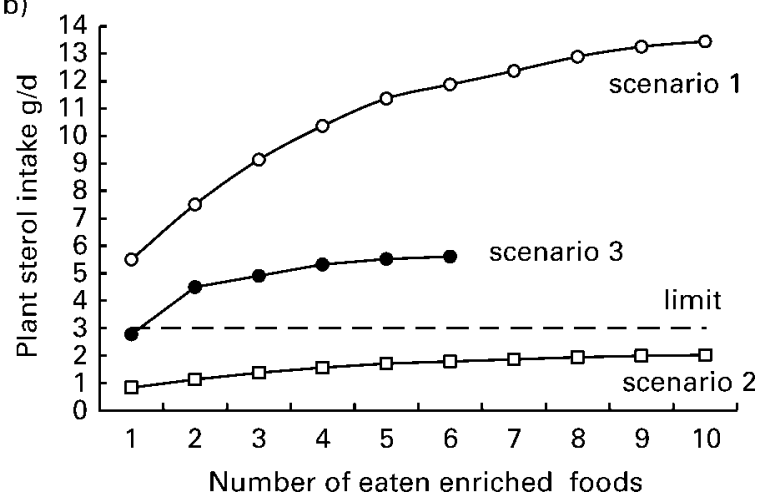

Fig. 2. Plant sterol intake by the consumption of multiple enriched food. The amounts were compared between the three scenarios along the mean intake (a) and along the 95th intake percentile (b). Each point represents the stepwise intake for the cumulated foods. The values at each point were based on the users of the plant sterol-enriched foods concerned. The ranking in the first and the second scenario is curd cheese, milk, hard cheese, margarine, whole grain bread, soft cheese, salami, yoghurt, liver sausage and processed cheese. In the third scenario the foods were cumulated in the following ranking: curd cheese, margarine, salami, yoghurt, liver sausage and processed cheese.

than $3 \mathrm{~g}$ phytosterols per day for each cumulation step for the third scenario.

\section{Discussion}

The data from the German NVS containing the $7 \mathrm{~d}$ weighed records from more than 23000 participants combined with the food composition table allows an assumption of the intake of food ingredients within greater consumer populations. Although the data in this study were collected in the western part of Germany before 1989 and some innovative food trends such as supplements, health drinks and designed snacks had not been launched at that time, the study gives a good overview on the consumption amounts and proportions of all common and many special foods. The NVS population study is suited for a German model population and can be used as a basic tool to carry out intake simulations and to create model calculations of food fortification, especially as the $7 d$ weighed record is thought to be the most accurate method of dietary assessment (Kroes et al. 2002). However, this method requires extraordinary effort over $7 \mathrm{~d}$ for the study participants. Underreporting via poor compliance and current conscious changes in nutrition behaviour can arise from this (Willet, 1998). (a)

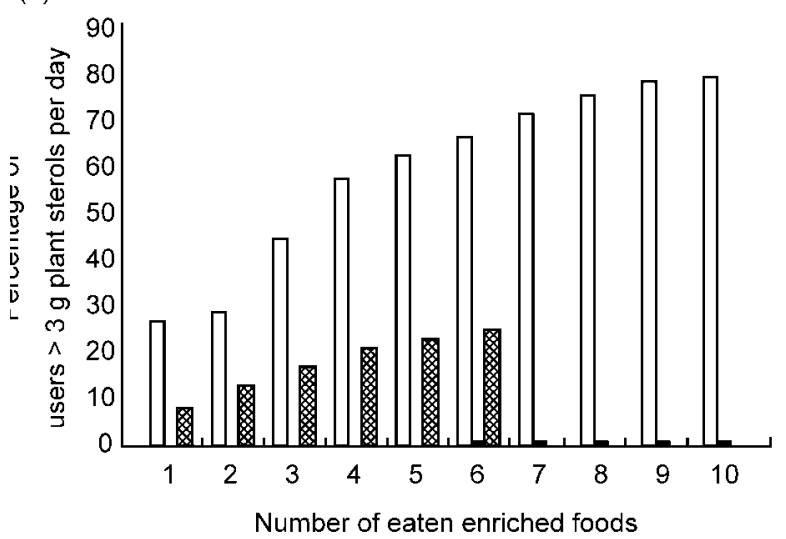

(b)

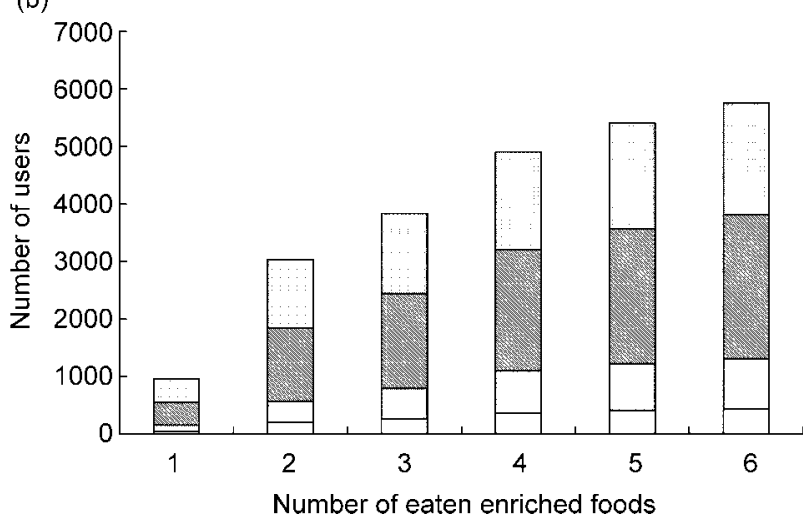

Fig. 3. (a), The percentages of users exceeding the recommended limit at each cumulation step in all scenarios $(\square$, scenario 1 ; $\mathbf{\square}$, scenario 2 ; $\otimes$, scenario 3). (b), Age pattern of this part of the users for each cumulation point according to the third scenario ( $\square, 4-14$ years; $\square, 5-24$ years; $\mathbb{\mathbb { N }}, 25-49$ years; 图, $>49$ years).

Based on the data of the National FINDIET 1997 Study with 2874 participants and using $24 \mathrm{~h}$ nutrition recalls, Raulio et al. (2001) calculated in model simulations the possible daily phytosterol/stanol intake by Finnish adults in four different age and sex cohorts. In this study, they exchanged one to four foods against their phytosterol-enriched counterparts in fifteen different food compositions. The enrichment concentrations were taken from existing Benecol ${ }^{\circledR}$-foodstuffs in Finland. The Finnish simulations resulted in phytosterol intake amounts from $2.3 \mathrm{~g}$ up to $10 \mathrm{~g} / \mathrm{d}$. The authors concluded that the daily intake of phytosterols can easily exceed $4 \mathrm{~g}$ and may even reach up to $9 \mathrm{~g}$ when different products are ingested.

Our simulation results affirm the intake dimension of the Finnish results. In addition, we found an intake satiation when all food servings were enriched with the same amount of plant sterols. We suppose that the satiation pattern reflects the daily consumption capacity for different food servings by individuals. Within our enrichment context the relation between plant sterol amount per proposed food serving in the context of the recommended intake maximum of $3 \mathrm{~g}$ plant sterols per day would allow only the adding of approximately $0.5 \mathrm{~g}$ plant sterols per food serving. Addition of $2 \mathrm{~g}$ plant sterols per proposed food serving could rise to an intake maximum of nearly $13 \mathrm{~g}$ plant sterols per day. Furthermore, the percentage of users taking excessive plant sterol amounts could increase to $80 \%$ 
(see Fig. 3). Our worst case simulation results are exemplary and depend on two parameters: accuracy in pooling different food varieties to one food type and the size of the food servings used as an allocation basis. Both parameters are of high empirical variance because there is no common agreement on an international standard for portion sizes and there is a tremendous diversity of foods on the European food market. Nevertheless, our maximum intake values are so far from $3 \mathrm{~g} /$ $\mathrm{d}$ that marginal variations of the preconditioned terms should produce comparable results.

The exclusive consumption of only one plant sterol-enriched food over a longer period will lead to a daily intake of an active dosage for $20-40 \%$ of the users concerned. This result corresponds with findings summarised in a Post Launch Monitoring (PLM) report on the consumption of a phytosterol-containing margarine presented by the applicant Unilever to the SCF (Scientific Committee on Food, 2002b). This PLM ascertained an average consumption of $1.2-1.4 \mathrm{~g}$ plant sterols per day (which corresponds to $15-18 \mathrm{~g}$ enriched margarine) by the study participants, although the ingestion of a daily serving of nearly $25 \mathrm{~g}$ margarine was recommended. The reason for these concordant observations may be that the defined servings of specific foods were not taken every day by most of the users. The average daily consumption amounts over $7 \mathrm{~d}$ were generally lower than the food servings which were actually eaten and which were exemplarily used as an allocation base for enrichment in this survey (see Table 2). The design of multiple plant sterol-containing foods for the same health-claim purpose appears reasonable in this circumstance. Indeed, this approach raised the problem of unintended excessive plant sterol intake by multiple dietary sources. As shown in our third scenario, it is possible to converge the enrichment context to the conflicting terms by selecting a limited number of appropriate foods as carrier foods for the plant sterol ingredient and by applying different enrichment amounts around the level of the active dosage. However, a major problem in this approach might be the definition of the features that make a food an appropriate carrier food for a functional ingredient, especially in a harmonised European food market.

The calculations in this study have been carried out in compliance with two assumptions, which are both afflicted with uncertainties. First, the consumers would take the plant sterolenriched foods in the same frequencies and amounts as the original non-fortified foods and second, the cholesterol-lowering claim and other labelling information would have no influence on the individual nutrition behaviour. In the case that the consumer ignores the labelling information, the first assumption would correspond to a worst case scenario of the plant sterol intake by multiple sources over time. Such an approach was also important for a previous enrichment topic, namely the defining of safe maximum added amounts for essential nutrients to foods. Flynn et al. (2003) proposed a model to calculate maximum amounts for safe adding of vitamins and minerals to foodstuffs, to minimise the risks of excessive intakes in individuals with high food consumption. The authors calculated the difference from a nutrient-specific tolerable upper intake level, a defined toxicological value not always available for nutrients (Walter, 2001), and the estimated nutrient intake by traditional nutrition in the 95th consumption percentile. Assuming that most people do not regularly eat more than $3600 \mathrm{kcal}(15072 \mathrm{~kJ})$ per day and that $50 \%$ of all relevant food products would be fortified with a specific nutrient, they calculated a safe enrichment amount per $100 \mathrm{kcal}(419 \mathrm{~kJ})$ of any food (Flynn et al. 2003). In contrast to our simulations, this model included the normal nutritional intake of micronutrients and used energy portions of $100 \mathrm{kcal}(419 \mathrm{~kJ})$ as an allocation base instead of proposed serving sizes. Certainly, food energy is a more objective parameter than experience-based serving sizes. However, it seems unlikely to be applicable to enriched beverages and supplements nearly free of energy. Additionally, the use of defined serving sizes as an allocation base in this simulation and also by phytosterol-food producers should facilitate the consumer's understanding of the consumed amount of the respective ingredient. Consumer's understanding is of the utmost importance because the excessive consumption of bioactive ingredients with dose-dependent effects bears even more unforseeable health risks compared to the intake of micronutrients. Furthermore, sufficient food composition and intake data as well as tolerable upper intake level values are not available for many bioactive substances at the time.

In contrast to the second assumption it should be expected that individual nutrition habits are determined in different degrees by rational expectations in which labelling information should be involved. Even though there is insufficient scientifically based knowledge about the influence of labelling information on consumers' nutrition habits (Kreuter et al. 1997), the individual consumption pattern may be strongly affected by actual health aspects (Miller \& Brown, 1999) as well as by imaginary ones. It should be anticipated that scientifically based and authorised health claims in connection with proposed serving sizes could have considerable influence on consumers' individual nutrition behaviour. Health claims and recommendations for consumption should ensure that consumers reach an active dosage with a serving of a specific functional food per day. Furthermore, such labelling information should also draw the consumer's attention to the recommended intake limit. Nevertheless, in view of the great potential to consume excessive amounts of bioactive ingredients via functional foods it seems reasonable to ensure consumer safety in a more impartial way. Health risks resulting from unknown interactions or interferences in the nutrient balance by long-term ingredient excesses in sensitive subgroups cannot be excluded. In the case of phytosterol intake, there are uncertainties about the long-term effects of the decreased concentrations of carotenes in the blood plasma of the users (Gylling et al. 1999; Hallikainen et al. 1999; Raeini-Sarjaz et al. 2002; Relas et al. 2001). In addition, Sudhop et al. (2002) recently discussed a moderate elevated plasma content of phytosterols as a potential risk factor in coronary heart diseases.

\section{Conclusions}

The health-claim triggered development of functional foods by adding bioactive ingredients to traditional foods seems to be a powerful instrument to reorganise the quantitative overall composition of human foodstuffs. Consequently, the overall intake amounts of such ingredients in different subgroups may obtain an unintended intake dimension over longer periods of time and may cause other than the desired effects on health. In the case of plant sterol compounds, the regular intake may increase from nearly $300 \mathrm{mg}$ by traditional nutrition up to a maximum of $13 \mathrm{~g} / \mathrm{d}$ by using a variety of plant sterol-enriched foods. Already the average daily phytosterol intake can easily reach quantities of 3-6g. That means that the prospective intake progression of phytosterols could rise from the 10 -fold amount in the intended 
purpose up to the approximately 50 -fold amount among individuals consuming high quantities and/or multiple enriched sources. Therefore, there is evidence to restrict the adding of active dosages of plant sterols only to a number of foods, easily manageable by consumers. Furthermore, the intake simulation framework containing well-characterised model populations, standardised food serving sizes and defined food selection criteria for modelling maintainable enrichment terms of functional ingredients should be qualified.

\section{References}

Adolf T, Schneider R, Eberhardt W, et al. (1995) Ergebnisse der Nationalen Verzehrsstudie (1985-1988) über die Lebensmittel- und Nährstoffaufnahme in der Bundesrepublik Deutschland. In VERASchriftenreihe Band XI (The German National Consumption Study (1985-89): Consumption of foods and nutrients in the Federal German Republic. in: VERA Study Volume XI) [W Kuebler, HJ Anders and W Heeschen, editors]. Niederkleen: Wissenschaftlicher Fachverlag Dr. Fleck.

AID-Auswertungs- und Informationsdienst für Ernährung, Landwirtschaft und Forsten e.V. (AID - Assessment and information service for Nutrition, Agriculture and Forestry) (1991) MONICA Mengenliste. AID Spezial 1, 1-41.

Ayesh R, Weststrate JA, Drewitt PN \& Hepburn PA (1999) Safety evaluation of phytosterol esters. Part 5. Faecal short-chain fatty acid and microflora content, faecal bacterial enzyme activity and serum female sex hormones in healthy normolipidaemic volunteers consuming a controlled diet either with or without a phytosterol ester-enriched margarine. Food Chem Toxicol 37, 1127-1138.

Baker VA, Hepburn PA, Kennedy SJ, Jones PA, Lea LJ, Sumpter JP \& Ashby J (1999) Safety evaluation of phytosterol esters. Part 1. Assessment of oestrogenicity using a combination of in vivo and in vitro assays. Food Chem Toxicol 37, 13-22.

Colling M, Weggemann S, Doring A, Keil U \& Wolfram G (1989) Nutrition survey of adults using a 7-day protocol - a pilot study in the Augsburg MONICA project. Offentl Gesundheitswes 51, 94-97.

Diplock AT, Aggett PJ, Ashwell M, Bornet F, Fern EB \& Roberfroid MB (1999) Scientific concepts of functional foods in Europe. Consensus document. Br J Nutr 81, Suppl. 1, S1-S27.

Flynn A, Moreiras O, Stehle P, Fletcher RJ, Muller DJ \& Rolland V (2003) Vitamins and minerals: a model for safe addition to foods. Eur J Nutr 42, 118-130.

Gylling H, Puska P, Vartiainen E \& Miettinen TA (1999) Retinol, vitamin $\mathrm{D}$, carotenes and alpha-tocopherol in serum of a moderately hypercholesterolemic population consuming sitostanol ester margarine. Atherosclerosis 145, 279-285.

Gylling H, Siimes MA \& Miettinen TA (1995) Sitostanol ester margarine in dietary treatment of children with familial hypercholesterolemia. J Lipid Res 36, 1807-1812.

Hallikainen MA, Sarkkinen ES \& Uusitupa MI (1999) Effects of low-fat stanol ester enriched margarines on concentrations of serum carotenoids in subjects with elevated serum cholesterol concentrations. Eur J Clin Nutr 53, 966-969.

Hallikainen MA, Sarkkinen ES \& Uusitupa MI (2000) Plant stanol esters affect serum cholesterol concentrations of hypercholesterolemic men and women in a dose-dependent manner. J Nutr 130, 767-776.

Hendriks HF, Weststrate JA, van Vliet T \& Meijer GW (1999) Spreads enriched with three different levels of vegetable oil sterols and the degree of cholesterol lowering in normocholesterolaemic and mildly hypercholesterolaemic subjects. Eur J Clin Nutr 53, 319-327.

Hepburn PA, Horner SA \& Smith M (1999) Safety evaluation of phytosterol esters. Part 2. Subchronic 90-day oral toxicity study on phytosterol esters - a novel functional food. Food Chem Toxicol 37, $521-532$.
Hornstra G, Barth CA, Galli C, et al. (1998) Functional food science and the cardiovascular system. Br J Nutr 80, Suppl. 1, S113-S146.

Klemm C, Mathis G, Christ M, et al. (1999) Der Bundeslebensmittelschlüssel (The German Food Code and Nutrient Base) (BLS II.3). Konzeption, Aufbau und Dokumentation der Datenbank blsdat (Conception, structure and documentation of the data-base blsdat), BGVV-Hefte $\mathbf{8}$, $5-45$.

Kreuter MW, Brennan LK, Scharff DP \& Lukwago SN (1997) Do nutrition label readers eat healthier diets? Behavioral correlates of adults' use of food labels. Am J Prev Med 13, 277-283.

Kroes R, Muller D, Lambe J, et al. (2002) Assessment of intake from the diet. Food Chem Toxicol 40, 327-385.

Law M (2000) Plant sterol and stanol margarines and health. BMJ 320, 861-864.

Mensink RP, Aro A, Den Hond E, German JB, Griffin BA, Ten Meer HU, Mutanen M, Pannemans D \& Stahl W (2003) PASSCLAIM - dietrelated cardiovascular disease. Eur J Nutr 42, Suppl. 1, 16-27.

Miller C \& Brown J (1999) Knowledge and use of the food label among senior women in the management of type 2 diabetes mellitus. $J$ Nutr Health Aging 3, 152-157.

Milner JA (2000) Functional foods: the US perspective. Am J Clin Nutr 71, Suppl. 6, 1654S-1659S, discussion 1674S-1675S.

Nguyen TT (1999) The cholesterol-lowering action of plant stanol esters. J Nutr 129, 2109-2112.

Ostlund RE Jr, McGill JB, Zeng CM, Covey DF, Stearns J, Stenson WF \& Spilburg CA (2002) Gastrointestinal absorption and plasma kinetics of soy Delta(5)-phytosterols and phytostanols in humans. Am J Physiol Endocrinol Metab 282, E911-E916.

Raeini-Sarjaz M, Ntanios FY, Vanstone CA \& Jones PJ (2002) No changes in serum fat-soluble vitamin and carotenoid concentrations with the intake of plant sterol/stanol esters in the context of a controlled diet. Metabolism 51, 652-656.

Raulio S, Nurttila A \& Mannonen L (2001) Adding phytosterols and -stanols to food - modelling the amount received by Finnish adults. Publications of National Food Agency 10, 3-22.

Relas H, Gylling H \& Miettinen TA (2001) Acute effect of dietary stanyl ester dose on post-absorptive alpha-tocopherol, beta-carotene, retinol and retinyl palmitate concentrations. $B r J$ Nutr $\mathbf{8 5}$, $141-147$.

Roberfroid MB (2000) Concepts and strategy of functional food science: the European perspective. Am J Clin Nutr 71, Suppl. 6, 1660S-1664S; discussion $1674 \mathrm{~S}-1675 \mathrm{~S}$.

Sanders DJ, Minter HJ, Howes D \& Hepburn PA (2000) The safety evaluation of phytosterol esters. Part 6 . The comparative absorption and tissue distribution of phytosterols in the rat. Food Chem Toxicol 38, $485-491$.

Scientific Committee on Food (2000) Opinion of the Scientific Committee on Food on the Request for the Safety Assessment of the Use of Phytosterol Esters in Yellow Fat Spreads. Brussels: European Commission.

Scientific Committee on Food (2002a) General View of the Scientific Committee on Food on the Long-term Effects of the Intake of Elevated Levels of Phytosterols from Multiple Dietary Sources, with Particular Attention to the Effects on $\beta$-carotene. Brussels: European Commission.

Scientific Committee on Food (2002b) Opinion of the Scientific Committee on Food on a Report on Post Launch Monitoring of 'Yellow Fat Spreads with Added Phytosterol Esters'. Brussels: European Commission.

Sudhop T, Gottwald BM \& von Bergmann K (2002) Serum plant sterols as a potential risk factor for coronary heart disease. Metabolism 51, $1519-1521$.

Tunstall-Pedoe H (1985) Monitoring trends in cardiovascular disease and risk factors: the WHO 'Monica' project. WHO Chron 39, $3-5$.

Waalkens-Berendsen DH, Wolterbeek AP, Wijnands MV, Richold M \& Hepburn PA (1999) Safety evaluation of phytosterol esters. Part 3. 
Two-generation reproduction study in rats with phytosterol esters - a novel functional food. Food Chem Toxicol 37, 683-696.

Walter P (2001) Towards ensuring the safety of vitamins and minerals. Toxicol Lett 120, 83-87.

Wester I (1999) Dose responsivness to plant stanol esters. Eur Heart J Suppl 1, Suppl. S, S104-S108.

Weststrate JA, Ayesh R, Bauer-Plank C \& Drewitt PN (1999) Safety evaluation of phytosterol esters. Part 4. Faecal concentrations of bile acids and neutral sterols in healthy normolipidaemic volunteers consuming a controlled diet either with or without a phytosterol ester-enriched margarine. Food Chem Toxicol 37, 1063-1071.

Weststrate JA \& Meijer GW (1998) Plant sterol-enriched margarines and reduction of plasma total- and LDL-cholesterol concentrations in normocholesterolaemic and mildly hypercholesterolaemic subjects. Eur $J$ Clin Nutr 52, 334-343.

Willet W (1998) Nutritional Epidemiology, 2nd ed. Oxford: Oxford University Press. 